\section{Check for updates}

Cite this: RSC Adv., 2017, 7, 24085

\title{
A silk fibroin hydrogel with reversible sol-gel transition
}

\author{
Zhuping Yin, ${ }^{a}$ Feng Wu, ${ }^{a}$ Tieling Xing, ${ }^{a}$ Vamsi K. Yadavalli, ${ }^{\text {b }}$ Subhas C. Kundu ${ }^{\mathrm{c}}$ \\ and Shenzhou Lu (D) *a
}

In this report, a novel silk fibroin hydrogel with a reversible sol-gel transition capacity is presented, in which the base material of this reversible hydrogel is hydrophilic silk fibroin (HSF) obtained by immersing a dried regenerated Bombyx mori silk fibroin (SF) condensate in deionized water (DI water) and collecting its lixivium. The resulting HSF sol can exhibit an enhanced sol-gel transition within several hours at suitable temperatures $\left(25-50{ }^{\circ} \mathrm{C}\right)$ without any exterior additive, even at extremely low concentrations $(<0.2 \%$, w $/$ v). The HSF gel can perform thixotropic, reversible gel-sol transitions triggered by a facile cycled shearthinning and resting procedure. Reversible sol-gel transition kinetics analyses and dynamic measurements of the micro-structure transformation during the transition demonstrate that both the $\beta$ sheet self-assembling process and metastable hydrogen bonding ( $\mathrm{H}$-bond) interactions among these large scale $\beta$-sheet aggregates play essential roles in the significant enhancement of the reversible HSF sol-gel transition. Due to the reversible, thixotropic sol-gel transitions and suitable viscoelasticity of its shear-thinning system for matching random sizes/shapes, the HSF system is possibly an alternative injectable hydrogel for applications in 3-dimensional (3D) cell culture and tissue repair in situ.

Received 6th March 2017

Accepted 6th April 2017

DOI: 10.1039/c7ra02682j

rsc.li/rsc-advances hydrogel can make the reversible sol-gel transitions with triggers and can easily be used for cell seeding or encapsulating and injection into randomly shaped/sized tissue defects. ${ }^{14,15}$ Although many self-healing reversible hydrogels have been reported, these materials require special triggers to initiate its sol-gel transition, such as a robust temperature change, significant $\mathrm{pH}$ decrease, distinct solvent polarity variation, even UV light exposure. ${ }^{16-22}$ Nevertheless, some of these robust triggers may cause an irritation to the separated sensitive cells and limit its practical use for cell culture and tissue repair. ${ }^{14}$ Moreover, the possible leakage of the initial low viscous solution to neighbouring tissue may occur even in a strong sol-gel transition dynamic. ${ }^{23}$

Recently, several new thixotropic, reversible hydrogels have emerged and enabled 3-dimensional cell culture and tissue repair in situ, such as polypeptides, hydroxypropylcellulose, PEO-PHB-PEO triblock copolymers (polyoxyethylene-polyhydroxybutyrate-polyoxyethylene) and their blending groups. These hydrogels can perform a reversible sol-gel transition via an easy cycled shear-thinning and resting process rather than robust, fatal chemical/physical triggers. ${ }^{24-31}$ These hydrogels exhibit a static semi-solid characteristic and can transform into a minimal viscoelastic fluid via a simple shear-thinning management.

The silk fibroin from Bombyx mori silkworms has been widely explored as biomaterials for decades due to its versatile strengths, including an apparent biocompatibility, controllable degradability and excellent mechanical properties. The silk 
fibroin can be easily processed into a variety of material formats (e.g., films, sponges, microspheres, electrospun fibres, nanoparticles, hydrogels) with mild aqueous-based processing. ${ }^{32-38}$ The SF hydrogel has drawn increasing attention in tissue repair, cell culture and drug delivery applications due to its outstanding biocompatibility and mechanical properties. ${ }^{39-43}$

However, as a stable dynamic and instable thermodynamic system, the sol-gel transition time of aqueous regenerated SF sol without exterior stimuli is quite long, usually a week to a month, ${ }^{4-46}$ which may limit its practical use. Therefore, quite a few physical and/or chemical methods have been used to stimulate and enhance the gelation kinetics of SF, including the physical methods of decreasing $\mathrm{pH}$, ultrasonic treatment, blending with polyhydric alcohol agents or ionic surfactants, and the chemical methods of chemical crosslinking or a polyreaction of additive micromolecule agents..$^{39,44-46}$ Nevertheless, these physically or chemically stimulated sol-gel transitions are almost irreversible.

Researchers have successfully designed and artificially produced a class of silk-like polypeptides using gene engineering techniques. These polypeptides can easily self-assemble into hydrogel from aqueous solution with physical or chemical triggers. ${ }^{13}$ Moreover, Liu, et al. has ever reported that the silk fibroin can be endowed with a reversible, thixotropic sol-gel performance by decreasing the hydrophility of surroundings to narrow the gap between solvent and solvend to unfold SF chains in micelles, via using the alcohol, sodium chloride and a facile centrifugation process, further resulting in a nanofibrils gel network structure. ${ }^{47}$ In addition, Bai, et al. also accomplished a reversible SF sol-gel transition by using a concentrationdilution method and incubating process at $60{ }^{\circ} \mathrm{C}$, in which the gel network can be circularly fractured into short nanofibers or nanoparticles and self-assemble into larger superamolecular aggregations via a facile cycled ultrasonic treatment and resting procedure, with absolutely no change of $\beta$-sheet contents. ${ }^{11}$

The mulberry silk fibroin consists of a heavy chain $\left(M_{\mathrm{w}} \sim 390\right.$ $\mathrm{kD})$ and a light chain $\left(M_{\mathrm{w}} \sim 26 \mathrm{kD}\right)$ that are chemically crosslinked by a disulfide bond, including regular hydrophobic, crystalline region sequences and irregular hydrophilic, noncrystalline region domains..$^{33,38,45}$ Although there are already some research about silk-based reversible, thixotropic gel system, ${ }^{\mathbf{1 3}, 47}$ but the reason why these reversible sol-gel characteristics come into being is still not fully understood and revealed. In this work, we made a thixotropic, reversible SF gel with respect to enhance inherent hydrophility of silk-like peptides to bridge the gap between silk-like peptides (solvend) and water (solvent) but not decrease the hydrophility of solvent. The relatively hydrophilic peptide sequences with a higher hydrophility than original SF are extracted from the regenerated aqueous Bombyx mori silk fibroin by immersing its dried condensates in deionized water. Then the hydrophilic silk fibroin (HSF) lixivium is collected and the insoluble residue is discarded. The results demonstrate that the HSF solution can easily undergo reversible, thixotropic sol-gel transitions within several hours triggered by facile cycled shear-thinning and resting managements, in the temperature range of $25-50{ }^{\circ} \mathrm{C}$ and even at extremely low concentration $(<0.2 \%)$. Due to the extraordinary reversible HSF sol-gel transition kinetics with no need of exterior additives and the proper viscoelasticity of its shear-thinning system, the HSF systems have potentials as injectable materials for $3 \mathrm{D}$ cell encapsulation, cell culture and tissue repair in situ.

\section{Experiment section}

\section{Preparation of silk fibroin solution}

The Bombyx mori cocoons were boiled three times in an aqueous solution of $0.05 \% \mathrm{Na}_{2} \mathrm{CO}_{3}$ for $30 \mathrm{~min}$ at $98-100{ }^{\circ} \mathrm{C}$. The boiled cocoons were rinsed with deionized (DI) water each time to remove the sericin on the surface of silk fibre. The rinsed cocoons were then dried at $60{ }^{\circ} \mathrm{C}$ for approximately $6 \mathrm{~h}$. To obtain the purified aqueous silk fibroin solution, the degummed and dried silk fibres were dissolved in aqueous lithium bromide solution (9.3 M LiBr) at $60{ }^{\circ} \mathrm{C}$ for $1 \mathrm{~h}$. Then, the dissolved and cooled silk fibroin solution with an approximate $10 \%$ concentration $(\mathrm{w} / \mathrm{v})$ was dialysed with running deionized water at $4{ }^{\circ} \mathrm{C}$ for 3 days using Slide-a-Lyzer dialysis cassettes (Pierce, MWCO 8-12 kD). The purified silk fibroin solution was centrifuged at $4500 \mathrm{rpm}$ for 10 minutes at $4{ }^{\circ} \mathrm{C}$ to remove insoluble and/or assembling aggregations. ${ }^{\mathbf{4 4 , 4 8 , 4 9}}$ The final silk fibroin concentration was approximately $5 \%(\mathrm{w} / \mathrm{v})$, measured by weighing the mass before and after a complete drying process.

\section{Preparation of hydrophilic silk fibroin solution}

The resulting purified silk fibroin solution $(5 \% \mathrm{w} / \mathrm{v})$ was poured in a polyethylene culture dish using a flowing cast method at $0.3 \mathrm{ml} \mathrm{cm}^{-2}$, followed by a drying process at $25{ }^{\circ} \mathrm{C}$ and $60 \%$ humidity for at least $24 \mathrm{~h}$. Then, the dried silk films were immersed in DI water for $1 \mathrm{~h}$ at $37^{\circ} \mathrm{C}$ in a water bath, with a SF condensates dissolving ratio less than $20 \%$. The immersion samples were centrifuged at $4500 \mathrm{rpm}$ for 5 minutes at $4{ }^{\circ} \mathrm{C}$ to precipitate the insoluble residual portion and collect the optically transparent and lyotropic hydrophilic silk fibroin lixivium. The concentration of the hydrophilic silk fibroin lixivium was approximately $0.80 \%(\mathrm{w} / \mathrm{v})$. Owing to its lyotropic characteristic, the soluble silk fibroin is called hydrophilic silk fibroin (HSF), while the insoluble part is called hydrophobic silk fibroin (ISF) herein.

\section{Sol-gel transition}

The HSF solution was diluted with DI water to $0.25 \%, 0.50 \%$, and $0.75 \%(\mathrm{w} / \mathrm{v})$ concentrations (a HSF concentration higher than $1.0 \%$ tend to result in a too robust HSF sol-gel transition within 1-2 hours) and cast into optically transparent sample bottles separately, followed by a resting process at temperatures of $4,25,37,50$, and $100{ }^{\circ} \mathrm{C}$. The sol-gel transition was determined when the sample vial appeared a slightly optically transparent white and did not fall from an inverted vial within $30 \mathrm{s.}^{\mathbf{3 9 , 4 0}}$

A Synergy HT Multi-Mode Microplate Reader (Bio-Tek Instruments, USA, ELISA) was also used to monitor and identify the sol-gel transition at $37{ }^{\circ} \mathrm{C}$ using a $550 \mathrm{~nm}$ visible light absorption of $1 \mathrm{ml} \mathrm{HSF}$ solution. The HSF sol was added to a 24- 
well plate and covered with $0.2 \mathrm{ml}$ tetradecane to avoid the evaporation of water, ${ }^{\mathbf{3 9}, \mathbf{4 4}}$ three parallel samples for each group.

To reveal the mechanism of the reversible, thixotropic solgel transition, the sol-gel transition kinetics of HSF/urea blending groups at different urea concentrations and a consistent HSF concentration were also measured using both of the methods mentioned above.

\section{Rheological measurements}

The rheological performance was tested with a Rheometer (AR2000, TA Instruments, New Castle, USA) at $37{ }^{\circ} \mathrm{C}$ equipped with a $20 \mathrm{~mm}$ cone plate $\left(\mathrm{Ti}, 20 / 1^{\circ}\right)$ and a Peltier temperature control system. A solvent trap and a low viscosity mineral oil were used to avoid water evaporation of the samples. The initial fresh HSF solution and the HSF systems before and after several cycles of shear-thinning processes using a MX-S/F adjustable vortex instrument were measured. The strain was set to $1 \%$ and the frequency sweep was collected over the angular frequency range of 0.1 to $100 \mathrm{rad} \mathrm{s}^{-1} \cdot{ }^{11,46}$

\section{Amino acid analysis}

The HSF solution is the lyotropic lixivium of dried SF condensates. Amino acid analysis was conducted to determine the difference between the HSF and its corresponding ISF after an immersion process. The HSF and ISF mentioned above were both dried at room temperature for $24 \mathrm{~h}$. The immersion times were set to 1 and $12 \mathrm{~h}$. To obtain the aqueous free amino acid solution, dried samples weighing approximately $100 \mathrm{mg}$ were thoroughly dissolved and hydrolysed in $10 \mathrm{ml}$ aqueous hydrochloric acid solution (GR, $6 \mathrm{~N}$ ) in an optically transparent vial, followed by an air elimination process with flowing $\mathrm{N}_{2}$ for $15 \mathrm{~s}$. The sealed sample vials were rested in a $110{ }^{\circ} \mathrm{C}$ environment for $24 \mathrm{~h}$. The cooled hydrochloric acid solution samples $(0.1 \mathrm{ml})$ were dried thoroughly in a $60{ }^{\circ} \mathrm{C}$ oven for $24 \mathrm{~h}$ and dissolved again in $0.02 \mathrm{~N} \mathrm{HCl}$ solution at a $40 \mathrm{mg} \mathrm{l}^{-1}$ concentration. The final solution was filtered using hydrophilic filter membranes with a $0.22 \mu \mathrm{m}$ pore dimension before its amino acid analysis using a L-8900 High Speed Amino Acid Analyzer. The mole percentage of the amino acid residues was calculated to determine the differences among these samples ( 3 parallel samples each group). The percentage differences between the HSF and ISF groups were measured using the following eqn (1):

Relative percentages difference (RPD, \%)

$$
=\frac{\mathrm{ISF}-\mathrm{HSF}}{\mathrm{ISF}} \times 100 \%
$$

where HSF is the mole percentage of one amino acid residue in the HSF group and ISF is the mole percentage of one amide acid residue in the ISF group corresponding to the HSF group.

\section{Surface tension testing (SFT)}

The Wilhemy plate method was used for surface tension measurement using a DataPhysics ${ }^{\mathrm{TM}}$ DCAT-21 surface tension instrument. The measurement was carried out in a circular thermostatted dish $\left(60 \mathrm{~cm}^{2}, 25 \pm 0.5{ }^{\circ} \mathrm{C}\right)$. The speed of the lift motor was $1.00 \mathrm{~mm} \mathrm{~s}^{-1}$ for detecting the surface of the liquid.
The results were determined when the standard deviation of the SFT was less than $0.03 \mathrm{mN} \mathrm{m}^{-1}$ in the last 50 measurement cycles and the accuracy was $0.001 \mathrm{mN} \mathrm{m}^{-1} \cdot{ }^{39}$ The HSF and SF solutions were prepared at $0.3 \%, 0.5 \%$, and $0.7 \%$ concentrations $(\mathrm{w} / \mathrm{v})$ for testing.

\section{Circular dichroism (CD)}

The CD spectra were used to monitor the macromolecule conformational transformation in HSF solution during the solgel transition. The CD spectra were recorded with a JASCO-815 spectrometer with a wavelength spectra range of 190-250 nm and a $1.0 \mathrm{~nm}$ wavelength step. The solution or gel samples were loaded in a sandwich quartz cell with a $0.1 \mathrm{~mm}$ path length. The results were the average accumulations of three measurements.

\section{Fluorescence measurements}

To monitor the hydrophilic-hydrophobic microenvironment changes and conformational transformation in HSF solution during the sol-gel transition, fluorescence measurement was performed using an Edinburgh Instrument FLS920 at $25{ }^{\circ} \mathrm{C}$. ${ }^{\mathbf{5 0}}$ The inherent tryptophan (Trp) contained in HSF was used as probes in the fluorescence measurement. The excitation wavelengths were set to $295 \mathrm{~nm}$, and the emission wavelength ranges were 300-500 nm, respectively. The excitation and emission slit widths were set to $2 \mathrm{~nm}$. The emission spectrum was recorded from the excitation of the sample with a $1 \mathrm{~cm}$ optical path.

\section{Zeta potentials and particle sizes}

A Nano-ZS90 particle sizer and a zeta potentiometer analyser (Malvern Instruments, UK) were used to record the particle size distribution and its corresponding zeta potential change in the HSF sol-gel transition at $37^{\circ} \mathrm{C}$. The $\mathrm{pH}$ of the HSF solution was maintained at $7.0 \pm 0.2$ during the entire testing period.

\section{Structure of HSF solution and gel}

The initial fresh HSF solution and its corresponding gel undergoing $24 \mathrm{~h}$ sol-gel transitions at 4,37 , and $100{ }^{\circ} \mathrm{C}$ were quickly frozen in liquid nitrogen and dried thoroughly in a $4{ }^{\circ} \mathrm{C}$ freeze dryer. The dried samples were cut into fine powder, which could easily travel through an 80 mesh sieve. Fourier transform infrared spectroscopy (FTIR) using a Nicolet 5700 FTIR (Nicolet Co., USA) was used to detect the conformation change after a potassium bromide $(\mathrm{KBr})$ pellet processing. The IR absorption spectra were recorded for the samples at 400$4000 \mathrm{~cm}^{-1}$. Raman spectra were also performed to reveal the conformation transitions of the samples using an XploRA Raman Spectrometer (Horiba JobinYvon, France) excited at a $532 \mathrm{~nm}$ wavelength. Raman scattering spectra were recorded in the region of $400-2000 \mathrm{~cm}^{-1}$ based on the average of 10 measurements. X-ray diffraction (XRD) was carried out to understand the aggregation structure of HSF using an X-ray diffractometer (X0 Pert-Pro MPD, PANalytical, Almelo, Holland) with $\mathrm{Cu} \mathrm{Ka}$ radiation. The XRD patterns were recorded in the region of $2 \theta$ from $5-45^{\circ}$ at a $10^{\circ} \mathrm{min}^{-1}$ speed, $40 \mathrm{kV}$ and $35 \mathrm{~mA}$. 


\section{Morphology of HSF hydrogel}

Field-emission scanning electron microscopy (FESEM, Hitachi, S4800, Japan) was used to observe the microstructures of the hydrogels after a freeze-drying processing. The freeze-dried samples were sputter coated with gold prior to imaging.

\section{Results}

\section{Rheological properties of the reversible HSF sol-gel system}

The HSF is extracted from the dried SF condensates by immersing them in DI water and collecting the optically transparent lixivium after a $4500 \mathrm{rpm}$ centrifuging process (Fig. 1). Interestingly, the HSF solution performs a significantly enhanced sol-gel transition kinetics within several hours without any exterior additive compared with the conventional SF sol-gel transition that usually takes a week to a month. ${ }^{\mathbf{4 4 4}}$ Moreover, the HSF gel can exhibit reversible gel-sol transitions triggered by facile cycled shear-thinning and resting managements, which is the typical characteristic of thixotropic gel (Fig. 1).

To measure the reversible characteristic in HSF sol-gel transition, steady rheological measurements were performed using a Rheometer (AR2000, TA Instruments, New Castle, USA) at $37{ }^{\circ} \mathrm{C}$ equipped with a $20 \mathrm{~mm}$ cone plate $\left(\mathrm{Ti}, 20 / 1^{\circ}\right)$ and a Peltier temperature control system. Fig. 2A and B show the optical morphologies and viscoelastic characteristics of the HSF and SF groups. The initial HSF and SF solution exhibit no remarkable difference in both their relatively low viscoelastic and optically transparent characteristics at a $0.5 \%(\mathrm{w} / \mathrm{v})$ concentration, corresponding to a homogeneously distributing micelle morphology with the micelle dimension of 20$200 \mathrm{~nm}$ (Fig. 2D). There are relatively obvious enhancements in optical absorption and viscoelastic characteristics of the HSF group after a 6 hour resting management at $37^{\circ} \mathrm{C}$, in contrast to those of the SF control group. Moreover, the storage modulus $G^{\prime}$ and the loss modulus $G^{\prime \prime}$ were collected as a function of frequency within the range of 0.1 to $100 \mathrm{rad} \mathrm{s}^{-1} \cdot{ }^{11,51}$ The $G^{\prime}$ is

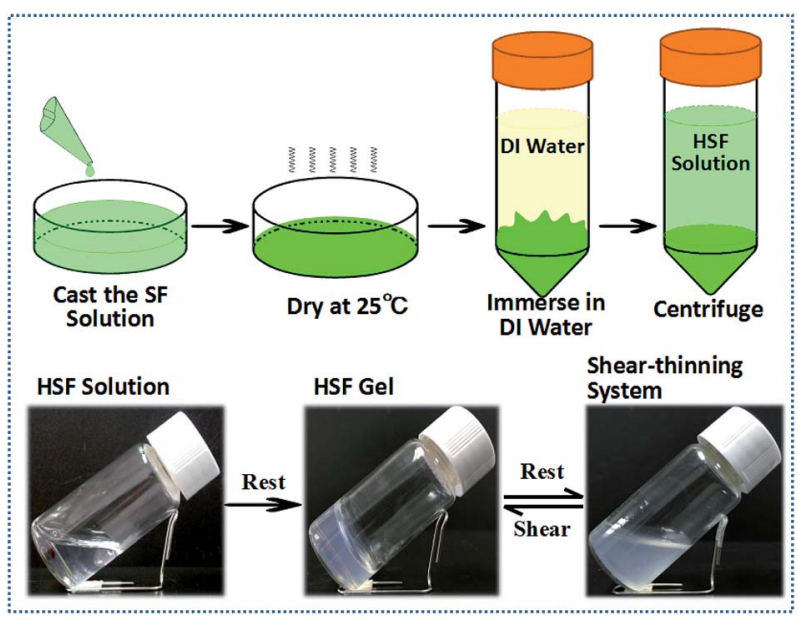

Fig. 1 Schematic representation of the reversible HSF gel fabrication process. larger than its corresponding $G^{\prime \prime}$ in all samples. There are significant increases (10-100 times) of the $G^{\prime}$ and $G^{\prime \prime}$ in HSF group and the $G^{\prime}$ is more than 10 times larger than $G^{\prime \prime}$ after its sol-gel transition (Fig. 2B), which is the representative viscoelastic symbol of hydrogel. ${ }^{11,51}$

To visualize the reversible, thixotropic sol-gel transition characteristic of HSF solution, the viscoelasticty of HSF systems were measured within 3 cycles of mechanical shear-thinning and resting managements. The semi-solid HSF gel undergoes a significant viscoelastic decrease and possesses a flowing morphology after each shear-thinning process (Fig. 2C and D). Both the $G^{\prime}$ and $G^{\prime \prime}$ of the flowing system can recover to values (1000-1100 Pa) similar to those of the initial HSF gel with the removal of shearing stress and a subsequent resting process, corresponding to a cycled morphology transition between relative closely connected mesoscale multilayer morphology and loosely connected multilayer structure (Fig. 2D), which is a representatively thixotropic, self-healing behavior.

The HSF shear-thinning sol $(0.5 \%, \mathrm{w} / \mathrm{v})$ was used as the "bioink" for a printing and patterning management to evaluate its injectable potentials using a biomedical injector. A coin patterned with a blooming chrysanthemum was used as the model for this patterning process. And the results indicate that the HSF shear-thinning sol can be easily used for injection and printing due to its relatively low viscoelasticty and result in a semi-solid gelatinous word (like "Silk") via sol-gel transitions. In addition, the HSF shear-thinning sol injected on the coin surface can well mimic the blooming chrysanthemum morphology after its sol-gel transition (Fig. 8).

The shear-thinning HSF solution shows a flowing but remarkable increments ( $\sim 10$ times larger) in its $G^{\prime}$ and $G^{\prime \prime}$ compared with the initial fresh HSF, corresponding to its optically oyster white colour, identical to those of the HSF gel but different from those of the initial optically transparent HSF solution (Fig. 2D). As evidence from the optically oyster white characteristic and mesoscale multilayer morphology of the HSF shear-thinning system, nearly identical to those of the initial HSF gel, we assume that the shear-thinning process do no damage to the mesoscale self-assembling aggregations in HSF gel but the physical interactions among these aggregation blocks/units, in agreement with the results of C. Yan and B. Ozbas. ${ }^{52,53}$ The initial homogeneous 20-200 nm micelles in HSF sol with minimal optical absorption can finally transform into a connected mesoscale multilayer morphology closely stacked with unilaminar nanoparticles (approximately 10-20 $\mathrm{nm}$ in diameter), corresponding to a HSF sol-gel transition. And there is a cycled mesoscale morphology transition between closely connected, larger multilayer structure and fractured multilayer units/blocks. Thus, the shear-thinning process makes no significant difference in HSF mesoscale multilayer units/blocks but the interaction among these multilayer aggregations (Fig. 2D). Presumably, there are weak but reversible physical interactions among HSF selfassembling aggregations in its gel, in which the physical interactions can be easily broken and regenerated during the shearing and resting (self-assembling) procedure.

FTIR, Raman spectra, and XRD were used to measure the conformational transition and aggregation structure of the HSF 

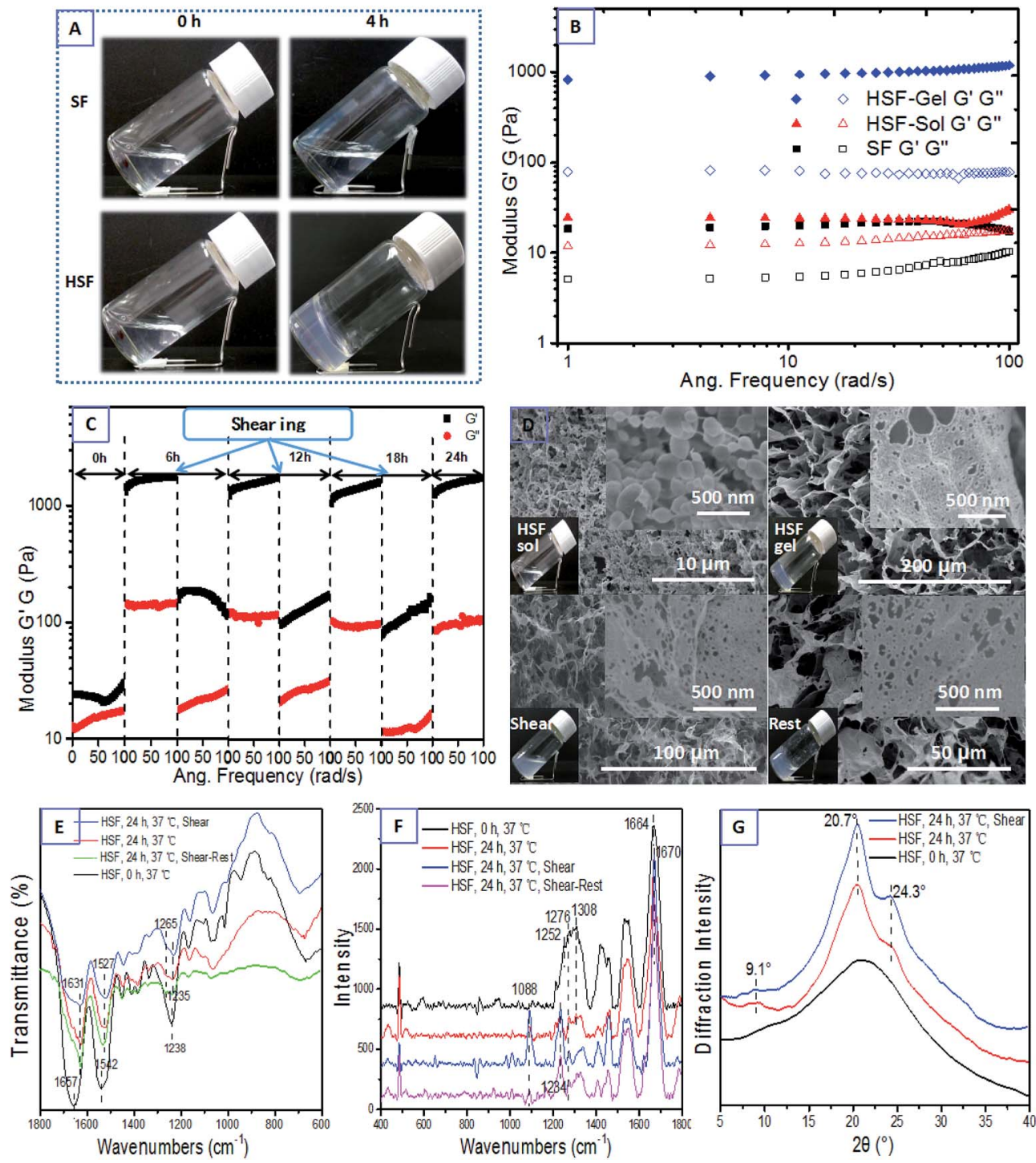

Fig. 2 Rheological characteristic and structure of the HSF sol-gel system, $37^{\circ} \mathrm{C}, 0.5 \%(\mathrm{w} / \mathrm{v})$ : (A) dynamic morphology of the $\mathrm{HSF}$ and $\mathrm{SF}$ sol, (B) storage modulus $\left(G^{\prime}\right)$ and loss modulus $\left(G^{\prime \prime}\right)$ of the initial HSF sol and gel compared with SF sol, (C) evolution of $G^{\prime}$ and $G^{\prime \prime}$ after several shearthinning to the HSF system, (D) morphology of reversible the HSF sol-gel system in SEM, (E)-(G) FTIR spectra, Raman spectra and XRD curves of HSF system.

systems herein. The initial HSF solution holds sharp infrared absorption peaks at $1657 \mathrm{~cm}^{-1}$ (amide I), $1542 \mathrm{~cm}^{-1}$ (amide II), and $1238 \mathrm{~cm}^{-1}$ (amide III), and Raman scatter peaks at 1664 $\mathrm{cm}^{-1}$ (amide I), $1308 \mathrm{~cm}^{-1}, 1276 \mathrm{~cm}^{-1}$ and $1252 \mathrm{~cm}^{-1}$ (amide II), corresponding to a reasonably widespread peak in the XRD curve (Fig. 2E-G), which shows a typical random coil conformation and amorphous aggregation structure. ${ }^{39,44,46-48}$ Interestingly, both the HSF gel, its shear-thinning system and selfhealing gel share identical peaks in the infrared absorption spectra, the Raman scatter and the XRD curves. The HSF gel, HSF shear-thinning and recovering systems exhibit infrared absorption peaks at $1631 \mathrm{~cm}^{-1}$ (amide I), $1527 \mathrm{~cm}^{-1}$ (amide II), 1265 and $1235 \mathrm{~cm}^{-1}$ (amide III), and Raman scatter peaks at $1670 \mathrm{~cm}^{-1}$ (amide I), $1234 \mathrm{~cm}^{-1}$ (amide III), and $1088 \mathrm{~cm}^{-1}$ (CC), corresponding to a representative Silk II crystal structure at $9.1^{\circ}, 20.7^{\circ}$, and $24.3^{\circ}$ positions. These are typical symbols of $\beta$ - sheet conformers and Silk II crystal structure. ${ }^{39,44,46-48}$ The results demonstrate that there is a significant conformational transformation from random coil to $\beta$-sheet conformation during the initial HSF sol-gel transition and the shear-thinning process is harmless to the $\beta$-sheet conformation and aggregation blocks/units in HSF gel, in agreement with the results from Bai, et al. ${ }^{11}$ Thus, the significant $\beta$-sheet assembly is not an essential factor for the self-healing recovery of the shearthinning HSF sol but some kinds of interactions easy to be broken and regenerated.

\section{Sol-gel transition kinetics of HSF}

The initial fresh HSF solution was obtained by the immersion way mentioned above (Fig. 1). The sol-gel transition was determined by the time point corresponding to half the stable 
optical absorbance at $550 \mathrm{~nm}$ detected in an ELISA and when the sample did not fall from an inverted vial within $30 \mathrm{s.} .^{39,40}$ The HSF solution can transform into a semi-solid hydrogel within several hours at a relatively low concentration (Fig. 3A), even lower than $0.2 \%(\mathrm{w} / \mathrm{v})$, while the gelation of normal aqueous $\mathrm{SF}$ solution usually takes a week to a month and can not be accomplished spontaneously with a SF concentration lower than $1 \%$. Moreover, there is a significant increasing sol-gel transition kinetics of HSF in positive proportion to its concentration (Fig. 3A), with a approximately 2 hours sol-gel transition time of the $1.5 \%$ concentrated HSF sol. A higher HSF concentration results in a stronger and faster interaction between the macromolecule chains and aggregations, similar to the gelation mechanism of normal regenerated SF or other polypeptides.

Surprisingly, a proper temperature range is essential to ensure the spontaneous HSF sol-gel transition within several hours (Table 1, Fig. 3E). The HSF solution performs a significantly increasing sol-gel transition kinetics within 2-6 h, positively correlated to the temperature in the range of $25-50{ }^{\circ} \mathrm{C}$. Remarkably, the HSF solution can hardly even cannot accomplish a sol-gel transition at relatively low or extremely high temperatures. The HSF solution at $4{ }^{\circ} \mathrm{C}$ shows an optically transparent and flowing characteristic for a long time $(>170 \mathrm{~h})$, arising from the difficulties to accomplish a $\beta$-sheet assembling in $4{ }^{\circ} \mathrm{C}$ (Fig. 3B-D). However, the HSF sol-gel transitions at $75{ }^{\circ} \mathrm{C}$ require quite a longer time than those at $25-50{ }^{\circ} \mathrm{C}$, according with a remarkable macroscopic oyster white turbid aggregations in its gel. The initial optically transparent HSF solution cannot transform into a homogeneous hydrogel but robustly aggregates and precipitates into a inhomogeneous oyster white turbid fluid with a high $\beta$-sheet content within 30 minutes at $100{ }^{\circ} \mathrm{C}$ (Fig. 3B-E). Presumably, the HSF sol-gel transition requires a proper assembling temperature to form
Table 1 The HSF gelation time at different temperatures ${ }^{a}$

\begin{tabular}{lllllll}
\hline Temperature $\left({ }^{\circ} \mathrm{C}\right)$ & 4 & 25 & 37 & 50 & 75 & 100 \\
Sol-gel time $(\mathrm{h})$ & $>170$ & 6 & 3 & 2 & $>10 \mathrm{~h}$ & -
\end{tabular}

${ }^{a}$ The HSF solution concentration is $0.75 \%(\mathrm{w} / \mathrm{v})$.

aggregations rich in $\beta$-sheets in addition to the relatively weak but essential interactions between these aggregations, such as loosely connected H-bonds, ${ }^{13}$ which cannot generate at $100{ }^{\circ} \mathrm{C}$.

To further uncover the mechanism of this sol-gel transition, the conformation and aggregation structure of HSF solution before and after a resting process at 4,37 , and $100{ }^{\circ} \mathrm{C}$ for $24 \mathrm{~h}$ were measured with FTIR, Raman spectroscopy, and XRD (Fig. 3B-D). The initial HSF solution shows several sharp infrared absorption peaks at $1659 \mathrm{~cm}^{-1}$ (amide I), $1540 \mathrm{~cm}^{-1}$ (amide II), and $1239 \mathrm{~cm}^{-1}$ (amide III), corresponding to the Raman scattering peaks at the $1664 \mathrm{~cm}^{-1}$ (amide I) and 1250 $\mathrm{cm}^{-1}$ (amide III). These are typical characteristics of random coil conformers. ${ }^{39,44,46-48}$ The initial HSF solution also shows an amorphous structure with a widespread peak at the XRD curve, similar to normal regenerated SF. After a culturing process at $4{ }^{\circ} \mathrm{C}$ for $24 \mathrm{~h}$, the HSF retains a flowing characteristic with conformation and aggregation structure similar to the initial HSF solution (Fig. 3B-E). In comparison with the nearly monotonous $\mathrm{HSF}$ group at $4{ }^{\circ} \mathrm{C}$, the $\mathrm{HSF}$ solution at $37{ }^{\circ} \mathrm{C}$ completely transform into a semi-solid gel within several hours, together with apparent micro-structure transitions from random coil to $\beta$-sheet conformers and amorphous state to Silk II crystal structure, ${ }^{\mathbf{3 9 , 4 4 , 4 6 - 4 8}}$ with sharp infrared absorption peaks at $1626 \mathrm{~cm}^{-1}$ (amide I), $1529 \mathrm{~cm}^{-1}$ (amide II), $1265 \mathrm{~cm}^{-1}$ and $1235 \mathrm{~cm}^{-1}$ (amide III), corresponding to the Raman scattering peaks at $1670 \mathrm{~cm}^{-1}$ (amide I), $1233 \mathrm{~cm}^{-1}$ (amide III) and typical
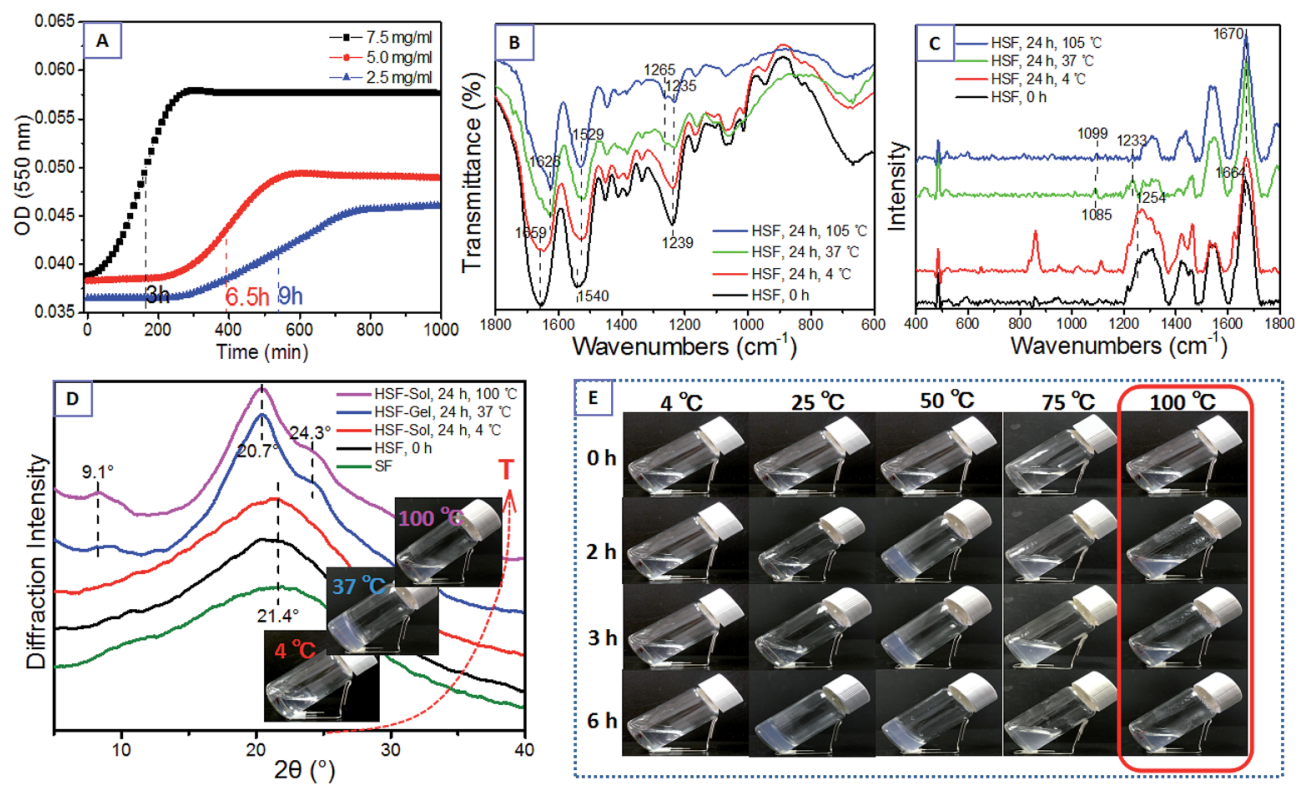

Fig. 3 Concentration/temperature dependence of HSF sol-gel transition kinetics: (A) dynamic optical absorption in the HSF sol-gel transition $\left(37^{\circ} \mathrm{C}\right)$, (B) FTIR spectra, (C) Raman spectra, (D) XRD curves, (E) dynamic optical morphology of the HSF solution resulting at different temperatures $(0.75 \%, w / v)$. 
Silk II crystal peaks at $9.1^{\circ}, 20.7^{\circ}$, and $24.3^{\circ}$ in the XRD curves (Fig. 3B-D). Surprisingly, the HSF solution resting at $100{ }^{\circ} \mathrm{C}$ for $24 \mathrm{~h}$ exhibits flowing and oyster white turbid fluid characteristics although a perfect $\beta$-sheet conformers and amorphous structure exist (Fig. 3B-E). The results reveal that both the weak physical interactions and $\beta$-sheet self-assembly process play vital roles in the robust HSF sol-gel transition. Thus, the aqueous HSF sol can hardly transform into gel arising from an extremely slow $\beta$-sheet assembling process at $4{ }^{\circ} \mathrm{C}$, while the HSF sol can not make homogeneous gels but result in oyster white turbid fluids within $30 \mathrm{~min}$ due to the robust $\beta$-sheet assembling and difficultly to fabricate some metastable interactions, like H-bonds, among these $\beta$-sheet aggregations.

The circular dichroism (CD) spectra were recorded at several time points in the HSF sol-gel transition (Fig. 4A). The initial HSF solution shows a low negative Cotton effect at approximately $201 \mathrm{~nm}$. The previous negative Cotton effect at $201 \mathrm{~nm}$ is subsequently replaced by a positive Cotton effect at $195 \mathrm{~nm}$ and a negative Cotton effect at $216 \mathrm{~nm}$ and $222 \mathrm{~nm}$ within the HSF sol-gel transition. This indicates a conformational transition from random coil to typical $\beta$-sheet and less $\beta$-turn conformers. $^{39,44,46-48}$ There are a more significant positive Cotton effect at $195 \mathrm{~nm}$ and a negative Cotton effect at $216 \mathrm{~nm}$ and $228 \mathrm{~nm}$ after a $12 \mathrm{~h}$ sol-gel transition.

The fluorescence probe technique has drawn much attention due to its sensitive fluorescence emission variations corresponding to its undergoing micro-environmental changes. ${ }^{50}$ To monitor the hydrophilic-hydrophobic micro-environmental change and conformational transformation that the HSF undergoes during the sol-gel transition, the interior contained tryptophan (Trp) in HSF was used as probe for fluorescence measurement.

The initial HSF solution exhibits a stronger interior fluorescence intensity than the control SF group at the same concentration, possibly resulting from a higher hydrophilic Trp content and more hydrophilic characteristic of HSF than SF group, ${ }^{50}$ according with the results obtained from amino acid analysis and SFT measurement (Fig. 7). Moreover, compared with the control SF group with no regular and remarkable interior fluorescent change, the HSF group shows a significant blue shift from $348 \mathrm{~nm}$ to $335 \mathrm{~nm}$ in its fluorescent emission peak. This is accompanied by a robust enhancement in its fluorescence intensity, especially in the 5-8 h time period, corresponding to the gelation time point $(6 \mathrm{~h})$ at $0.5 \% \mathrm{HSF}$ concentration (w/v) and $37{ }^{\circ} \mathrm{C}$ mentioned above (Fig. 3A). The results confirm that there is an apparent $\beta$-sheet assembly process hydrophobic micro-environment during the HSF solgel transition. ${ }^{50}$

Urea is a reagent with high $\mathrm{P}$ type electron pair contents that interacts with $\mathrm{H}$ atoms and fabricates $\mathrm{H}$-bonds easily, which is a proper inhibitor for fabrication of $\mathrm{H}$-bond interactions within the $\beta$-sheet HSF aggregations. ${ }^{54,55}$ To further elucidate the mechanism of the HSF sol-gel transition, the urea was blended with HSF at $0.04,0.08$, and $0.12 \mathrm{mg} \mathrm{ml}^{-1}$ concentrations and the final HSF concentrations were set to $0.2 \%$ and $0.4 \%(\mathrm{w} / \mathrm{v})$. The sol-gel transitional kinetics of the HSF/urea blending system was monitored using a ELISA instrument at $37^{\circ} \mathrm{C}$. Fig. 5 confirm that the higher the concentration of urea, the lower the HSF sol-gel transition kinetics. The HSF/urea blending system with a final HSF concentration of $0.2 \%$ was added to several optically transparent vials to visualize its sol-gel transition at 25, 37, and $50{ }^{\circ} \mathrm{C}$. Only the pure HSF group exhibits a sol-gel transition within 4-16 h, in proportion to the surrounding temperature in the $25-50{ }^{\circ} \mathrm{C}$ range. Moreover, the shear-thinning HSF sol blending with urea can hardly accomplish a sol-gel transition. Therefore, H-bond interaction plays a critical role in the selfhealing characteristic of HSF gel.

The Nano-ZS90 particle sizer and a zeta potentiometer analyser were used to visualize the dynamic particle size distribution and its corresponding zeta potential variation during the HSF sol-gel transition. Coinciding with the HSF sol-gel transition kinetics, a robust particle size increment occurs in the HSF system from the average size of $3 \mathrm{~nm}$ to hundreds and even thousands of nanometers within several hours, corresponding to a more negative zeta potential than those in normal SF gelation process, even lower than $-32 \mathrm{mV}$ (Fig. 6). The results indicate that the predominant negatively charged amino acid residues tend to be arranged on the surface of the self-assembling aggregations during the sol-gel transition, which make weak and loosely connected physical interaction, like $\mathrm{H}$-bond, easier to generated among these polar particles, ${ }^{\mathbf{1 1}}$ further resulting in the reversible HSF sol-gel transition.
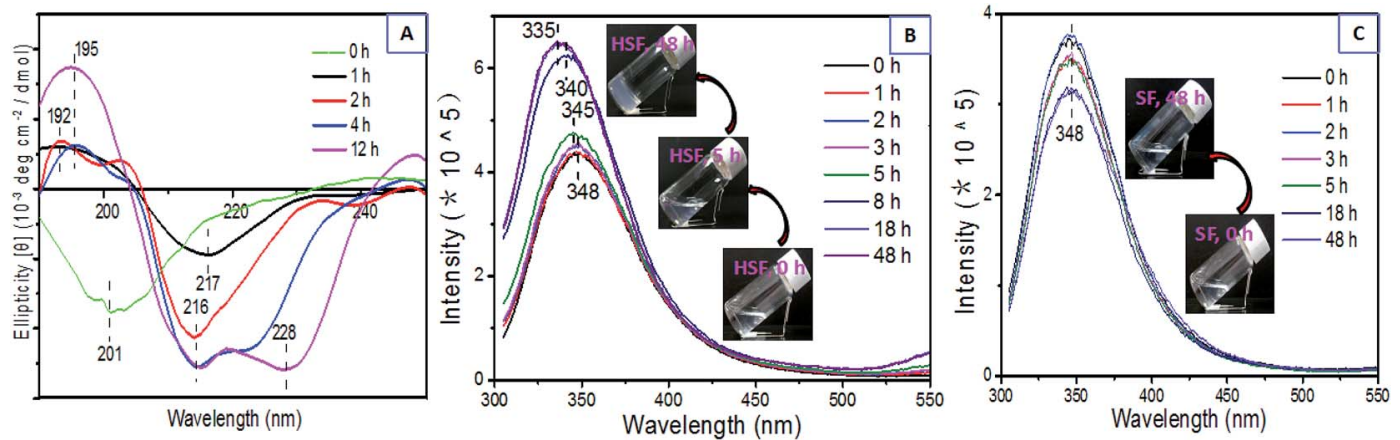

Fig. 4 Dynamic characteristics within the HSF sol-gel transition $\left(37^{\circ} \mathrm{C}\right)$ : (A) dynamic conformation structure from $\mathrm{CD}(0.75 \%, \mathrm{w} / \mathrm{v}),(\mathrm{B})$ interior fluorescence effect of Trp in the HSF $(0.50 \%, w / v),(C)$ interior fluorescence effect of $\operatorname{Trp}$ in the SF $(0.50 \%, w / v)$. 

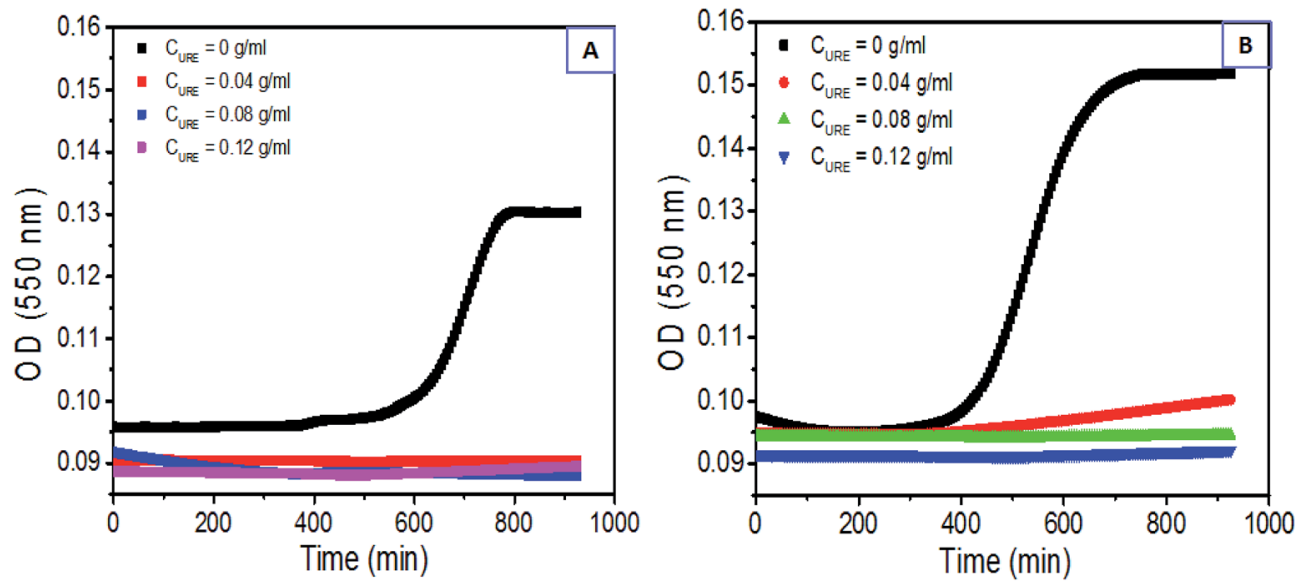

Fig. $5 \mathrm{H}$-bond dependence of HSF sol-gel transition kinetics: $(\mathrm{A})$ and $(\mathrm{B})$ dynamic optical absorption of HSF/urea blending system with $\mathrm{C}_{\mathrm{HSF}}=$ $0.2 \%$ and $0.4 \%$.
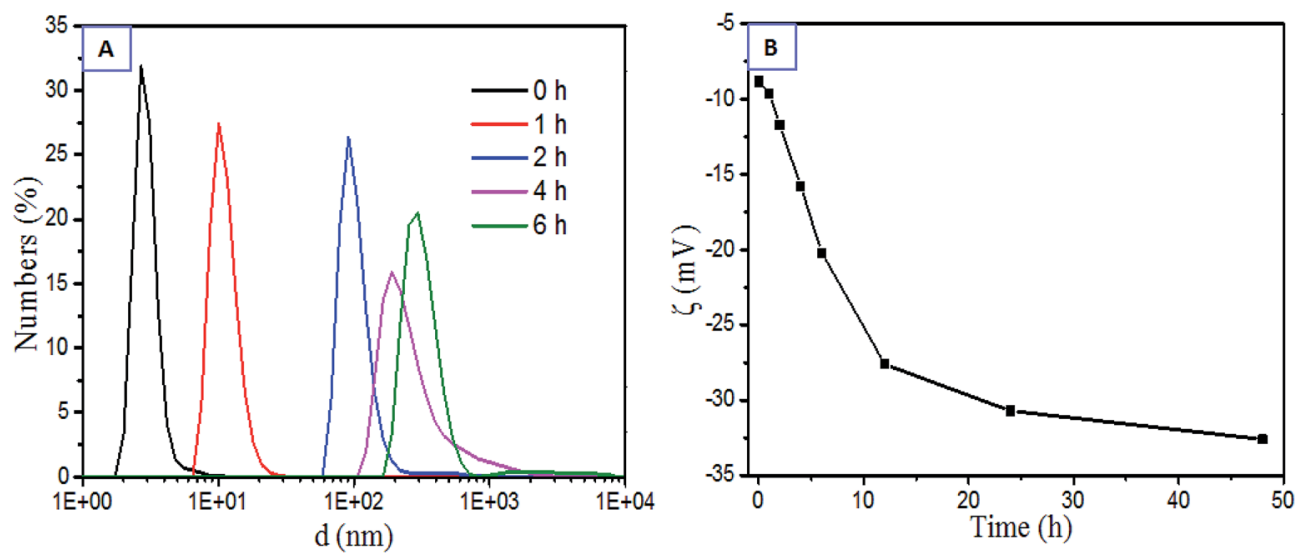

Fig. 6 Characteristics of aggregations within the $\mathrm{HSF}$ sol-gel transition at $37^{\circ} \mathrm{C}(0.5 \%, \mathrm{w} / \mathrm{v})$ : (A) dynamic particle size distribution, (B) dynamic zeta potential.

\section{Characteristics of the initial fresh HSF solution}

The silk fibroin is composed of a heavy chain $\left(M_{\mathrm{w}} \sim 390 \mathrm{kD}\right)$ and a light chain $\left(M_{\mathrm{w}} \sim 26 \mathrm{kD}\right)$ chemically crosslinked by a disulphide bond, including regular crystalline region sequences and irregular noncrystalline region domains. ${ }^{33,38,44,45,49}$ However, all these complicated polypeptide chains contain relatively constant basic units, 20 amino acid residues. The order and content of these 20 amino acid residues with special hydrophilic or hydrophobic side-chains play a critical, inherent role in polypeptides conformers in water. ${ }^{44,50}$

The HSF solution is the lyotropic, hydrophilic lixivium from SF condensates, obtained by immersing the dried SF films in DI water and collecting the optically transparent lixivium (Fig. 1). To figure out the reason why the enhanced and reversible solgel transition exists, amino acid analysis was carried out. This provides us the differences between lyotropic HSF and its corresponding insoluble residues (ISF) with respect to the composition of amino acid residues. The immersion times were set to 1 and 12 hours. The differences of amino acid residue contents between the HSF and ISF were exhibited with the top nine amide acids in SF polypeptides, which compose at least $95 \%$ molar content of the SF. The hydropathic index was used to evaluate the hydrophilic-hydrophobic ability of these amino acid residues (Fig. 7B). ${ }^{56}$ The amino acid residues with negative hydropathic index are relative hydrophilic than those with positive hydropathic index. ${ }^{56}$ Therefore, the results indicate that the HSF solution has higher hydrophilic content and lower hydrophobic amide acid content than the ISF solution, except for an abnormal tyrosine (Tyr) that mainly exists in the crystal sequence of SF (Fig. 7A). ${ }^{50}$ In conclusion, the HSF is the silk-like polypeptides with higher hydrophility than SF.

The surface tension instrument was also used to measure the characteristics of HSF and SF solution. The SFT of DI water is $71.98 \mathrm{mN} \mathrm{m}^{-1}$, in good agreement with the value obtained in a previous study.$^{39}$ Both the addition of HSF and SF result in an obvious decrease in the SFT of DI water. However, the SFT of HSF solutions are remarkably higher than SF solutions and exhibit a positive correlation with its concentration in the concentration (w/v) range of $0.3-0.7 \%$ (Fig. $7 \mathrm{C}$ ). This is a typical characteristic of hydrophilic reagents, in contrast to the SF, 

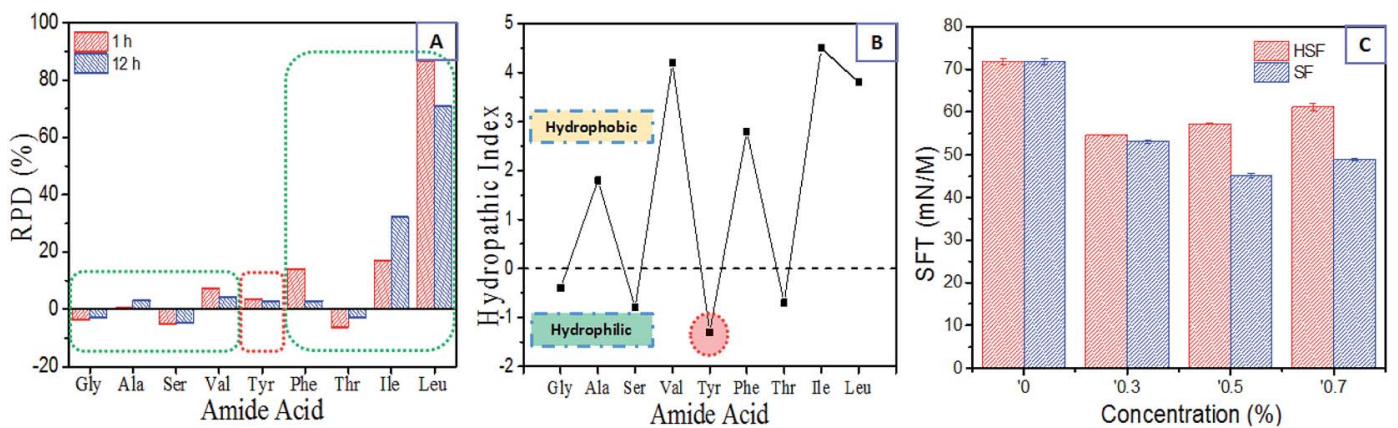

Fig. 7 Characteristics of the HSF and SF solution: (A) comparison in its predominant amino acid residues between the HSF and its corresponding residues, (B) hydropathic index to the predominant amide acid in SF, and (C) SFT of the HSF and SF solution.

according with its higher contents of hydrophilic amino acid residues mentioned above.

It's believed that the heavy chains of silk fibroin perform a micelle morphology (Fig. 2D) in an aqueous surrounding with its hydrophilic domains arranged on the surface of micelles and hydrophobic sequences distributed randomly inside the micelles, which make the $\beta$-sheet assembling among hydrophobic blocks from different micelles extremely difficult in accordance with a relatively slow sol-gel transition. ${ }^{57}$ Presumably, a higher hydrophilic amino acid contents make the HSF be relatively more hydrophilic compared to water (solvent) than normal SF, further resulting in a more extended/unfolded HSF chain structure in water, which is easy for $\beta$-sheet assembling and subsequent sol-gel transitions.

\section{Discussion}

Both the $\beta$-sheet assembling process and the metastable physical H-bond interactions play essential roles in the enhanced HSF sol-gel transition kinetics and its thixotropic, reversible sol-gel transition under the facile cycled shear-thinning and resting procedure (Fig. 8). The HSF polymer, lyotropic lixivium of a dried SF condensate (Fig. 1), is the relative hydrophilic polypeptides from silk fibroin (SF) with an average $3.0 \mathrm{~nm}$ particle size in DI water, in contrast to a $12.7 \mathrm{~nm}$ particle size in normal aqueous SF sol (Fig. 7A, B and 6A). The HSF tend to perform a relatively extended/unfolded conformation structure in aqueous solution and exhibit a higher SFT than normal regenerated aqueous SF solution at identical concentrations (Fig. 5C) owing to its better hydrophility comparable to water than those of normal SF sol, which results in the exposure of hydrophobic domains in HSF. Subsequently, the unfolded HSF chains can effortlessly $\beta$-sheet self-assemble into aggregations with typical Silk II crystal structure within several hours, corresponding to the transition from an initial homogeneous and optically transparent state to an oyster white and turbid characteristic (Fig. 4B). This silk fibroin hydrophily increasing method is similar to the self-assembly enhancing process in Liu, et al. via decreasing the surrounding hydrophily undergoing with the addition of alcohol to make the amphipathy of solvend and solvent comparable. Polar, hydrophilic peptide sequences are arranged on the surface of the HSF aggregations, resulting in a dynamic negative zeta potential (even lower than
$-32 \mathrm{mV}$ ) with the growth of the aggregation's dimension (Fig. 6). Consequently, the metastable H-bond interactions alternatively and easily self-fabricate among these hydrophilic polypeptide sequences arranged on the surface of the $\beta$-sheet aggregations (Fig. 8). This creates the final mesoscale HSF gel skeleton/network (Fig. 2D) and the final transition from the turbid HSF solution to the homogeneous HSF gel. Due to HSF sol-gel transition resulting from the synthetic effects of the $\beta$ sheet self-assembly process and the metastable H-bond interactions among the HSF assembling aggregations, a proper temperature range $\left(25-50{ }^{\circ} \mathrm{C}\right)$ is essential for HSF gelation (Fig. 3E and Table 1). The initial HSF solution usually takes a week to undergo a sol-gel transition at $4{ }^{\circ} \mathrm{C}$ (Table 1) and cannot accomplish a remarkable $\beta$-sheet self-assembly process in a short time (Fig. 3B). Hence, the HSF solution at $4{ }^{\circ} \mathrm{C}$ exhibits an optically transparent and flowing characteristic for a week (Fig. 3E and Table 1), corresponding to a typical random coil conformers, an amorphous aggregation structure and

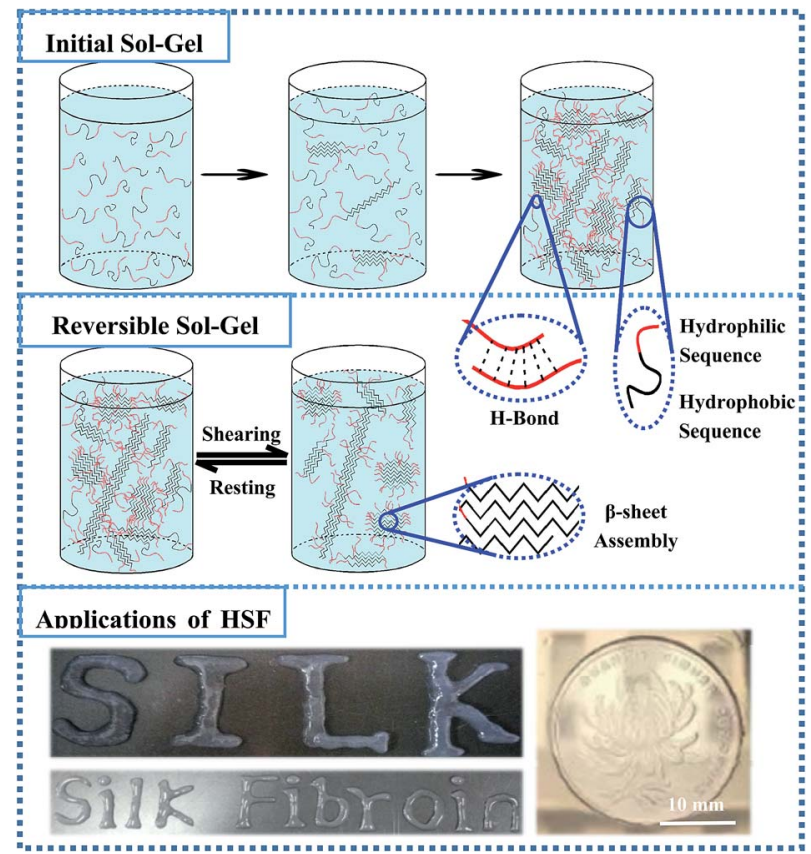

Fig. 8 Schematic of reversible thixotropic HSF sol-gel transition process. 
a homogeneous nanoscale micelle morphology (Fig. 3B-E and $2 \mathrm{D})$. However, the robustly enhanced $\beta$-sheet self-assembly process at $100{ }^{\circ} \mathrm{C}$ cannot endow the HSF sol-gel transition but an inhomogeneous turbid and oyster white liquid morphology because an extremely high temperature environment inhibits the fabrication of metastable $\mathrm{H}$-bond interactions among $\beta$-sheet aggregations (Fig. 3B-E). Reasonably, a higher HSF concentration induces a stronger HSF sol-gel transition kinetics and a shorter HSF gelation time (Fig. 3A) because of the more prominent $\beta$-sheet assembling processes and $\mathrm{H}$-bond interactions among these $\beta$-sheet aggregations.

Notably, HSF can accomplish a significantly enhanced solgel transition at an extremely low concentration (Fig. 3A), even lower than $0.2 \%(\mathrm{w} / \mathrm{v})$, whereas the normal regenerated $\mathrm{SF}$ solution can hardly transform into a homogeneous gel spontaneously at concentrations less than $2.0 \%(\mathrm{w} / \mathrm{v})$. This indicates the HSF sol can undergo an extraordinary sol-gel transition at an relatively lower concentration and molecule weight than the SF solution, which further confirms that the HSF domains overlap each other more easily due to its inherent hydrophilic characteristic and unfolded/extended conformation structure. ${ }^{58}$

Interestingly, the metastable, loosely packed $\mathrm{H}$-bond interactions among the $\beta$-sheet aggregations are easily broken and regenerated via the facile cycled mechanical shearing and resting procedure but not the strong interactions inside the closely packed $\beta$-sheet aggregations (Fig. 2), corresponding to a reversible sol-gel transition. These make the oyster white, turbid HSF shear-thinning system significantly lower viscoelasticity and a fractured, similar shaped/sized multilayer morphology compared with HSF gel (Fig. 2C and D). The sol-gel transition can be accomplished repeatedly due to the selfhealing, loosely-stacked $\mathrm{H}$-bond binding among the stable $\beta$ sheet aggregations, after a resting process (Fig. 2C, D and 8). Thus, the HSF gel network can be fractured into blocks/units ( $\beta$ sheet aggregations) under a shearing stress, resulting in a flowing oyster white turbid morphology. These blocks can be held together and transformed into a semi-solid gel via fabrication of the metastable, loosely-stacked H-bond interactions among the polar sequences on the surface of these aggregates, after removal of the shear stress and a resting process (Fig. 2C, D and 8), similar to the reversible gel network reported by Bai, et al. Due to the proper viscoelasticty and reversible sol-gel transition kinetics of HSF shear-thinning sol, it can be easily administrated for a printing and patterning applications via a injection process, resulting in some particular shaped/sized patterns of the HSF gel (Fig. 8). Because of the enhanced reversible HSF sol-gel transition kinetics without any exterior additive, and its shear-thinning system viscoelasticity that is properly suitable for clinical usage, the HSF systems have potentials as an injectable material for 3D cell culture and tissue repair in situ (Fig. 8).

\section{Conclusions}

The aqueous HSF solution, lyotropic lixivium of dried SF condensates, with a higher hydrophilic amino acid residue content and hydrophility, can easily and thoroughly transform into hydrogel within several hours at relatively low concentration (even lower than $0.2 \%$ ) and proper temperature $\left(25-50{ }^{\circ} \mathrm{C}\right.$ ). The HSF gel performs a remarkable, reversible thixotropic gelsol transition upon a facile cycled shearing and resting procedure. The results demonstrate that both the robust $\beta$-sheet selfassembly process and the metastable, loosely-stacked H-bond interactions among the polar charged aggregations due to the hydrophilic HSF sequences arranged on the surface of $\beta$-sheet aggregations play critical roles in the HSF reversible, thixotropic Gel-Sol transition. The reversible HSF sol-gel transition kinetics, proper viscoelasticity of HSF shear-thinning systems and gel based on a natural biomaterial make it acceptable as an injectable hydrogel for $3 \mathrm{D}$ cell culture and repair of defective tissues.

\section{Acknowledgements}

The work is supported by National Natural Science Foundation of China (Grant No. 51373114), PAPD and College Nature Science Research Project of Jiangsu Province, China (Grant No. 15KJA540001). SCK presently holds an ERA Chair Full Professor position at the 3B's Research Group, University of Minho, Portugal, and is supported by the European Union Framework Programme for Research and Innovation Horizon 2020 under grant agreement no 668983 - FoReCaST.

\section{References}

1 J. C. Bragg, H. Kweon, Y. Y. Jo, J. L. Kwang and C. C. Lin, In situ formation of silk-gelatin hybrid hydrogels for affinitybased growth factor sequestration and release, RSC Adv., 2016, 115, 114353-114360.

2 J. L. Drury and D. J. Mooney, Hydrogels for tissue engineering: scaffold design variables and applications, Biomaterials, 2003, 24, 4337-4351.

3 N. A. Peppas, J. Z. Hilt, A. Khademhosseini and R. Langer, Hydrogels in biology and medicine: from molecular principles to bionanotechnology, Adv. Mater., 2006, 18, 1345-1360.

4 S. M. Potter and T. B. DeMarse, A new approach to neural cell culture for long-term studies, J. Neurosci. Methods, 2001, 110, 17-24.

5 S. Rodin, A. Domogatskaya, S. Ström, E. M. Hansson, K. R. Chien, J. Inzunza, O. Hovatta and K. Tryggvason, Long-term self-renewal of human pluripotent stem cells on human recombinant laminin-511, Nat. Biotechnol., 2010, 28, 611-615.

6 P. Lu, V. M. Weaver and Z. Werb, The extracellular matrix: a dynamic niche in cancer progression, J. Cell Biol., 2012, 196, 395-406.

7 N. H. Romano, D. Sengupta, C. Chung and S. C. Heilshorn, Protein-engineered biomaterials: nanoscale mimics of the extracellular matrix, Biochim. Biophys. Acta, Gen. Subj, 2011, 1810, 339-349.

8 D. D. McKinnon, D. W. Domaille, T. E. Brown, K. A. Kyburz, E. Kiyotake, J. N. Cha and K. S. Anseth, Measuring cellular 
forces using bis-aliphatic hydrazone crosslinked stressrelaxing hydrogels, Soft Matter, 2014, 10, 9230-9236.

9 R. V. Rughani, M. C. Branco, D. J. Pochan and J. P. Schneider, De novo design of a shear-thin recoverable peptide-based hydrogel capable of intrafibrillar photopolymerization, Macromolecules, 2010, 43, 7924-7930.

$10 \mathrm{~L}$. Yu and J. Ding, Injectable hydrogels as unique biomedical materials, Chem. Soc. Rev., 2008, 37, 1473-1481.

11 S. Bai, X. Zhang, Q. Lu, W. Sheng, L. Liu, B. Dong, D. L. Kaplan and H. Zhu, Reversible hydrogel-solution system of silk with high beta-sheet content, Biomacromolecules, 2014, 15, 3044-3051.

12 H. Meng, J. Zheng, X. Wen, Z. Cai, J. Zhang and T. Chen, pHand Sugar-Induced Shape Memory Hydrogel Based on Reversible Phenylboronic Acid-Diol Ester Bonds, Macromol. Rapid Commun., 2015, 36, 533-537.

13 W. H. Rombouts, D. W. de Kort, T. T. H. Pham, C. P. van Mierlo, M. W. Werten, F. A. de Wolf and J. van der Gucht, Reversible Temperature-Switching of Hydrogel Stiffness of Coassembled, Silk-Collagen-Like Biomacromolecules, 2015, 16, 2506-2513.

$14 \mathrm{H}$. Wang and S. C. Heilshorn, Adaptable hydrogel networks with reversible linkages for tissue engineering, Adv. Mater., 2015, 27, 3717-3736.

15 Y. N. Wei, Q. Q. Wang, T. T. Gao, M. Kong, K. K. Yang, Y. An, S. Y. Jiang, J. Li, X. J. Cheng and X. G. Chen, 3-D culture of human umbilical vein endothelial cells with reversible thermosensitive hydroxybutyl chitosan hydrogel, J. Mater. Sci.: Mater. Med., 2013, 24, 1781-1787.

16 X. Qiu, Y. Yang, L. Wang, S. Lu, Z. Shao and X. Chen, Synergistic interactions during thermosensitive chitosan- $\beta$ glycerophosphate hydrogel formation, RSC Adv., 2011, 2, 282-289.

17 A. M. Kloxin, A. M. Kasko, C. N. Salinas and K. S. Anseth, Photodegradable hydrogels for dynamic tuning of physical and chemical properties, Science, 2009, 324, 59-63.

18 Z. Zhang, Y. Liu, X. Chen and Z. Shao, Multi-responsive polyethylene-polyamine/gelatin hydrogel induced by noncovalent interactions, RSC Adv., 2016, 54, 48661-48665.

19 T. F. Scott, A. D. Schneider, W. D. Cook and C. N. Bowman, Photoinduced plasticity in cross-linked polymers, Science, 2005, 308, 1615-1617.

20 B. M. Gillette, J. A. Jensen, M. Wang, J. Tchao and S. K. Sia, Dynamic Hydrogels: Switching of 3D Microenvironments Using Two-Component Naturally Derived Extracellular Matrices, Adv. Mater., 2010, 22, 686-691.

21 M. C. Roberts, M. C. Hanson, A. P. Massey, E. A. Karren and P. F. Kiser, Dynamically restructuring hydrogel networks formed with reversible covalent crosslinks, Adv. Mater., 2007, 19, 2503-2507.

22 X. Chen, M. A. Dam, K. Ono, et al., A thermally re-mendable cross-linked polymeric material, Science, 2002, 295(5560), 1698-1702.

23 R. N. Shah, N. A. Shah, M. M. D. R. Lim, C. Hsieh, G. Nuber and S. I. Stupp, Supramolecular design of self-assembling nanofibers for cartilage regeneration, Proc. Natl. Acad. Sci. U. S. A., 2010, 107, 3293-3298.
24 L. Haines-Butterick, K. Rajagopal, M. Branco, D. Salick, R. Rughani, M. Pilarz, M. S. Lamm, D. J. Pochan and J. P. Schneider, Controlling hydrogelation kinetics by peptide design for three-dimensional encapsulation and injectable delivery of cells, Proc. Natl. Acad. Sci. U. S. A., 2007, 104, 7791-7796.

25 C. Yan, A. Altunbas, T. Yucel, R. P. Nagarkar, J. P. Schneider and D. J. Pochan, Injectable solid hydrogel: mechanism of shear-thinning and immediate recovery of injectable $\beta$ hairpin peptide hydrogels, Soft Matter, 2010, 6, 5143-5156.

26 B. D. Olsen, J. A. Kornfield and D. A. Tirrell, Yielding behavior in injectable hydrogels from telechelic proteins, Macromolecules, 2010, 43, 9094-9099.

27 Q. Wang, L. Wang, M. S. Detamore and C. Berkland, Biodegradable colloidal gels as moldable tissue engineering scaffolds, Adv. Mater., 2008, 20, 236-239.

28 Y. S. Pek, A. C. Wan, A. Shekaran, L. Zhuo and J. Y. Ying, A thixotropic nanocomposite gel for three-dimensional cell culture, Nat. Nanotechnol., 2008, 3, 671-675.

29 J. Li, X. Li, X. Ni, X. Wang, H. Li and K. W. Leong, Selfassembled supramolecular hydrogels formed by biodegradable PEO-PHB-PEO triblock copolymers and $\alpha$ cyclodextrin for controlled drug delivery, Biomaterials, 2006, 27, 4132-4140.

30 D. Gupta, C. H. Tator and M. S. Shoichet, Fast-gelling injectable blend of hyaluronan and methylcellulose for intrathecal, localized delivery to the injured spinal cord, Biomaterials, 2006, 27(11), 2370-2379.

31 C. T. S. W. P. Foo, J. S. Lee, W. Mulyasasmita, A. Parisi-Amon and S. C. Heilshorn, Two-component protein-engineered physical hydrogels for cell encapsulation, Proc. Natl. Acad. Sci. U. S. A., 2009, 106, 22067-22072.

32 D. Kaplan, W. W. Adams, B. Farmer and C. Viney, Silk polymers: materials science and biotechnology, American Chemical Society, Washington, DC, 1994.

33 C. Vepari and D. L. Kaplan, Silk as a biomaterial, Prog. Polym. Sci., 2007, 32, 991-1007.

34 N. Panda, A. Bissoyi, K. Pramanik and A. Biswas, Development of novel electrospun nanofibrous scaffold from $P$. ricini and $A$. mylitta silk fibroin blend with improved surface and biological properties, Mater. Sci. Eng., $C$, 2015, 48, 521-532.

35 A. Motta, L. Fambri and C. Migliaresi, Regenerated silk fibroin films: thermal and dynamic mechanical analysis, Macromol. Chem. Phys., 2002, 203, 1658-1665.

36 Z. Shao and F. Vollrath, Materials: surprising strength of silkworm silk, Nature, 2002, 418, 741-741.

37 H. J. Kim, U. J. Kim, G. Vunjak-Novakovic, B. H. Min and D. L. Kaplan, Influence of macroporous protein scaffolds on bone tissue engineering from bone marrow stem cells, Biomaterials, 2005, 26, 4442-4452.

38 D. N. Rockwood, R. C. Preda, T. Yücel, X. Wang, M. L. Lovett and D. L. Kaplan, Materials fabrication from Bombyx mori silk fibroin, Nat. Protoc., 2011, 6, 1612-1631.

39 X. Wu, J. Hou, M. Li, J. Wang, D. L. Kaplan and S. Lu, Sodium dodecyl sulfate-induced rapid gelation of silk fibroin, Acta Biomater., 2012, 8, 2185-2192. 
40 J. Ming, M. Li, Y. Han, Y. Chen, H. Li, B. Zuo and F. Pan, Novel two-step method to form silk fibroin fibrous hydrogel, Mater. Sci. Eng., C, 2016, 59, 185-192.

41 B. Balakrishnan and R. Banerjee, Biopolymer-based hydrogels for cartilage tissue engineering, Chem. Rev., 2011, 111(8), 4453-4474.

42 H. Wu, S. Liu, L. Xiao, X. Dong, Q. Lu and D. L. Kaplan, Injectable and $\mathrm{pH}$-Responsive Silk Nanofiber Hydrogels for Sustained Anticancer Drug Delivery, ACS Appl. Mater. Interfaces, 2016, 8, 17118-17126.

43 M. Fini, A. Motta, P. Torricelli, G. Giavaresi, N. N. Aldini, M. Tschon and C. Migliaresi, The healing of confined critical size cancellous defects in the presence of silk fibroin hydrogel, Biomaterials, 2005, 26, 3527-3536.

44 A. Matsumoto, J. Chen, A. L. Collette, U. J. Kim, G. H. Altman, P. Cebe and D. L. Kaplan, Mechanisms of silk fibroin sol-gel transitions, J. Phys. Chem. B, 2006, 110, 21630-21638.

45 S. Kapoor and S. C. Kundu, Silk protein-based hydrogels: promising advanced materials for biomedical applications, Acta Biomater., 2016, 31, 17-32.

46 Z. Gong, Y. Yang, Q. Ren, X. Chen and Z. Shao, Injectable thixotropic hydrogel comprising regenerated silk fibroin and hydroxypropylcellulose, Soft Matter, 2012, 8, 2875-2883.

47 Y. Liu, S. Ling, S. Wang, X. Chen and Z. Shao, Thixotropic silk nanofibril-based hydrogel with extracellular matrix-like structure, Biomater. Sci., 2014, 2, 1338-1342.

48 S. Nagarkar, T. Nicolai, C. Chassenieux and A. Lele, Structure and gelation mechanism of silk hydrogels, Phys. Chem. Chem. Phys., 2010, 12, 3834-3844.

49 S. Lu, J. Li, S. Zhang, Z. Yin, T. Xing and D. L. Kaplan, The influence of the hydrophilic-lipophilic environment on the structure of silk fibroin protein, J. Phys. Chem. B, 2015, 3, 2599-2606.
50 I. Georgakoudi, I. Tsai, C. Greiner, C. Wong, J. DeFelice and D. L. Kaplan, Intrinsic fluorescence changes associated with the conformational state of silk fibroin in biomaterial matrices, Opt. Express, 2007, 15, 1043-1053.

51 L. Zhang, J. Qian, Y. Fan, W. Feng, Z. Tao and H. Yang, A facile $\mathrm{CO}_{2}$ switchable nanocomposite with reversible transition from sol to self-healable hydrogel, RSC Adv., 2015, 5, 62229-62234.

52 B. Ozbas, J. Kretsinger, K. Rajagopal, J. P. Schneider and D. J. Pochan, Salt-triggered peptide folding and consequent self-assembly into hydrogels with tunable modulus, Macromolecules, 2004, 37, 7331-7337.

53 C. Yan, A. Altunbas, T. Yucel, R. P. Nagarkar, J. P. Schneider and D. J. Pochan, Injectable solid hydrogel: mechanism of shear-thinning and immediate recovery of injectable $\beta$ hairpin peptide hydrogels, Soft Matter, 2010, 6, 5143-5156.

54 J. H. Woychik, J. A. Boundy and R. J. Dimler, Starch gel electrophoresis of wheat gluten proteins with concentrated urea, Arch. Biochem. Biophys., 1961, 94, 477-482.

55 S. P. Fling and D. S. Gregerson, Peptide and protein molecular weight determination by electrophoresis using a high-molarity tris buffer system without urea, Anal. Biochem., 1986, 155, 83-88.

56 J. Kyte and R. F. Doolittle, A simple method for displaying the hydropathic character of a protein, J. Mol. Biol., 1982, 157, 105-132.

57 Q. Lu, H. Zhu, C. Zhang, F. Zhang, B. Zhang and D. L. Kaplan, Silk self-assembly mechanisms and control from thermodynamics to kinetics, Biomacromolecules, 2012, 13, 826-832.

58 R. H. Colby, Structure and linear viscoelasticity of flexible polymer solutions: comparison of polyelectrolyte and neutral polymer solutions, Rheol. Acta, 2010, 49, 425-442. 